\title{
Existence Theorem about Triple Positive Solutions for a Boundary Value Problem with p-Laplacian
}

\section{Bo Sun}

School of Statistics and Mathematics, Central University of Finance and Economics, Beijing, China

Email: sunbo19830328@163.com

How to cite this paper: Sun, B. (2018) Existence Theorem about Triple Positive Solutions for a Boundary Value Problem with p-Laplacian. Journal of Applied Mathematics and Physics, 6, 29-35.

https://doi.org/10.4236/jamp.2018.61004

Received: November 13, 2017

Accepted: January 2, 2018

Published: January 5, 2018

\begin{abstract}
In this paper, by applying a fixed point theorem to verify the existence of at least three positive solutions to a three-point boundary value problem with $\mathrm{p}$-Laplacian. The interesting point is the nonlinear term is involved with the first-order derivative explicitly.
\end{abstract}

\section{Keywords}

Positive Solutions, Boundary Value Problem, p-Laplacian

\section{Introduction}

In this paper, we will consider the positive solutions to the following three-point boundary value problem with p-Laplacian

$$
\begin{gathered}
\left.\left(\phi_{p}\left(u^{\prime}(t)\right)\right)^{\prime}+f\left(t, u(t), u^{\prime}(t)\right)\right)=0, t \in(0,1), \\
u(0)=0, \quad u(1)=u(\eta),
\end{gathered}
$$

where $\phi_{p}(s)=|s|^{p-2} s, \quad p>1, \quad \eta \in(0,1)$ is a constant and $f(t, x, y) \in C([0,1] \times[0,+\infty) \times R \rightarrow[0,+\infty))$.

The study of positive solutions on second-order boundary value problems for ordinary differential equations has aroused extensive interest, one may see [1]-[10] and references therein.

Among the substantial number of works dealing with nonlinear differential equations we mention the boundary value problem (1) and (2). One thing to be mentioned is that nonlinear ter $\mathrm{f}$ is involved with the first-order derivative explicitly.

\section{Preliminaries}

Firstly, we present here some necessary definitions and background material of 
the theory of cones in ordered Banach spaces.

Definition 2.1. Let $E$ be a real Banach space. A nonempty closed set $P \subset E$ is said to be a cone provided that

1) $a u+b v \in P$ for all $u, v \in P$ and all $a \geq 0, b \geq 0$, and

2) $u,-u \in P$ implies $u=0$.

Definition 2.2. The map $\psi$ is said to be a nonnegative continuous concave functional on $P$ provided that $\psi: P \rightarrow[0, \infty)$ is continuous and

$$
\psi(t x+(1-t) y) \geq t \psi(x)+(1-t) \psi(y)
$$

for all $x, y \in P$ and $t \in[0,1]$. Similarly, we say the map $\alpha$ is a nonnegative continuous convex functional on $P$ provided that $\alpha: P \rightarrow[0, \infty)$ is continuous and

$$
\alpha(t x+(1-t) y) \leq t \alpha(x)+(1-t) \alpha(y)
$$

for all $x, y \in P$ and $t \in[0,1]$.

Definition 2.3. Let $r>a>0, L>0$ be constants, $\psi$ is a nonnegative continuous concave functional and $\alpha, \beta$ are nonnegative continuous convex functionals on the cone $P$. Define the following convex sets

$$
\begin{aligned}
& P(\alpha, r ; \beta, L)=\{u \in P \mid \alpha(u)<r, \beta(u)<L\}, \\
& \bar{P}(\alpha, r ; \beta, L)=\{u \in P \mid \alpha(u) \leq r, \beta(u) \leq L\}, \\
& P(\alpha, r ; \beta, L ; \psi, a)=\{u \in P \mid \alpha(u)<r, \beta(u)<L, \psi(u)>a\}, \\
& \bar{P}(\alpha, r ; \beta, L ; \psi, a)=\{u \in P \mid \alpha(u) \leq r, \beta(u) \leq L, \psi(u) \geq a\} .
\end{aligned}
$$

The following assumptions as regards the nonnegative continuous convex functions $\alpha, \beta$ are used

$\left(B_{1}\right)$ : there exists $M>0$ such that $\|x\| \leq M \max \{\alpha(x), \beta(x)\}$, for all $x \in P$;

$\left(B_{2}\right): P(\alpha, r ; \beta, L) \neq \varnothing$, for any $r>0, L>0$.

Next, we present a fixed point theorem established in [10], in which Bai and Ge generalized the Leggett-Williams' fixed point theorem. The generalization is achieved by introducing on cone of continuous functionals satisfying certain properties. The technique using functionals to replace norms has been proved very useful in generalizing some fixed point theorems.

Theorem 2.1. [10] Let $E$ be a Banach space, $P \subset E$ is a cone and $r_{2} \geq d>b>r_{1}>0, L_{2} \geq L_{1}>0$. Assume the $\alpha, \beta$ are nonnegative continuous convex functionals satisfying $\left(B_{1}\right)$ and $\left(B_{2}\right), \psi$ is a nonnegative concave functional on $P$, such that

$$
\psi(y) \leq \alpha(y) \text { for all } y \in \bar{P}\left(\alpha, r_{2} ; \beta, L_{2}\right)
$$

and $T: \bar{P}\left(\alpha, r_{2} ; \beta, L_{2}\right) \rightarrow \bar{P}\left(\alpha, r_{2} ; \beta, L_{2}\right)$ is a completely continuous operator. Suppose that

$\left(C_{1}\right):\left\{y \in \bar{P}\left(\alpha, d ; \beta, L_{2} ; \psi, b\right) \mid \psi(y)>b\right\} \neq \varnothing, \quad \psi(T y)>b$ for all $y \in \bar{P}\left(\alpha, d ; \beta, L_{2} ; \psi, b\right)$,

$\left(C_{2}\right): \alpha(T y)<r_{1}, \quad \beta(T y)<L_{1}$, for all $y \in \bar{P}\left(\alpha, r_{1} ; \beta, L_{1}\right)$,

$\left(C_{3}\right): \psi(T y)>b$ for all $y \in \bar{P}\left(\alpha, r_{2} ; \beta, L_{2} ; \psi, b\right)$ with $\alpha(T y)>d$.

Then $T$ has at least three fixed points $y_{1}, y_{2}, y_{3} \in \bar{P}\left(\alpha, r_{2} ; \beta, L_{2}\right)$ with 


$$
\begin{aligned}
y_{1} & \in P\left(\alpha, r_{1} ; \beta, L_{1}\right), \\
y_{2} & \in\left\{\bar{P}\left(\alpha, r_{2} ; \beta, L_{2} ; \psi, b\right) \mid \psi(y)>b\right\}, \\
\text { and } \quad y_{3} & \in \bar{P}\left(\alpha, r_{2} ; \beta, L_{2}\right) \backslash\left(\bar{P}\left(\alpha, r_{2} ; \beta, L_{2} ; \psi, b\right) \cup \bar{P}\left(\alpha, r_{1} ; \beta, L_{1}\right)\right) .
\end{aligned}
$$

\section{Multiple Positive Solutions for (1) and (2)}

Let the Banach space $E=C^{1}[0,1]$ be endowed with the norm

$$
\|u\|:=\max \left\{\max _{0 \leq t \leq 1}|u(t)|, \max _{0 \leq t \leq 1}\left|u^{\prime}(t)\right|\right\} .
$$

and define the cone $P \subset E$ by

$$
P=\{u \in E \mid u(t) \geq 0, u(0)=0, u \text { is concave on }[0,1]\} .
$$

Choose a natural number $k \geq \max \left\{\frac{1}{\eta}, \frac{2}{1-\eta}\right\}$. For notational convenience, we denote

$$
M=\frac{p-1}{p} \min \left\{\left(\frac{1+\eta}{2}-\frac{1}{k}\right)^{\frac{p}{p-1}},\left(\eta-\frac{1}{k}\right)^{\frac{p}{p-1}}+\left(1-\frac{1}{k}-\frac{1+\eta}{2}\right)^{\frac{p}{p-1}}\right\}, \quad N=\frac{p-1}{p} .
$$

Define functionals

$$
\alpha(u)=\max _{0 \leq t \leq 1}|u(t)|, \quad \beta(u)=\max _{0 \leq t \leq 1}\left|u^{\prime}(t)\right|, \quad \psi(u)=\min _{1 / k \leq t \leq 1-(1 / k)}|u(t)| \text {, for } u \in P .
$$

Then $\alpha, \beta: P \rightarrow[0,+\infty)$ are nonnegative continuous convex functionals satisfying $\left(B_{1}\right)$ and $\left(B_{2}\right)$, and $\psi$ is nonnegative continuous concave functional on $P$, it is also clear that $\psi(u) \leq \alpha(u)$ for all $u \in P$.

Lemma 3.1. For $y \in L[0,1]$ then the boundary value problem

$$
\left\{\begin{array}{l}
\left(\phi_{p}\left(u^{\prime}(t)\right)\right)^{\prime}+y(t)=0, \quad t \in(0,1), \\
u(0)=0, \quad u(1)=u(\eta)
\end{array}\right.
$$

has a unique solution

$$
u(t)=\left\{\begin{array}{l}
\int_{0}^{t} \phi_{p}^{-1}\left(\int_{s}^{\sigma} y(r) d r\right) d s, \quad 0 \leq t \leq \sigma, \\
\int_{0}^{\eta} \phi_{p}^{-1}\left(\int_{s}^{\sigma} y(r) d r\right) d s+\int_{t}^{1} \phi_{p}^{-1}\left(\int_{\sigma}^{s} y(r) d r\right) d s, \quad \sigma \leq t \leq 1 .
\end{array}\right.
$$

where $\sigma_{u} \in[\eta, 1]$ is the solution of the equation

$$
\int_{\eta}^{x} \phi_{p}^{-1}\left(\int_{s}^{x} y(r) d r\right) d s=\int_{x}^{1} \phi_{p}^{-1}\left(\int_{x}^{s} y(r) d r\right) d s .
$$

Proof. The proof can be obtained by regular calculation, so we omit it here. We define an operator $T: P \rightarrow E$ by

$$
(T u)(t)=\left\{\begin{array}{l}
\int_{0}^{t} \phi_{p}^{-1}\left(\int_{s}^{\sigma} f\left(r, u(r), u^{\prime}(r)\right) d r\right) d s, \quad 0 \leq t \leq \sigma, \\
\int_{0}^{\eta} \phi_{p}^{-1}\left(\int_{s}^{\sigma} f\left(r, u(r), u^{\prime}(r)\right) d r\right) d s+\int_{t}^{1} \phi_{p}^{-1}\left(\int_{\sigma}^{s} f\left(r, u(r), u^{\prime}(r)\right) d r\right) d s, \quad \sigma \leq t \leq 1 .
\end{array}\right.
$$

where $\sigma_{u} \in[\eta, 1]$ is the solution of the equation

$$
\int_{\eta}^{x} \phi_{p}^{-1}\left(\int_{s}^{x} f\left(r, u(r), u^{\prime}(r)\right) d r\right) d s=\int_{x}^{1} \phi_{p}^{-1}\left(\int_{x}^{s} f\left(r, u(r), u^{\prime}(r)\right) d r\right) d s .
$$

by Lemma 3.1, we know that boundary value problem (1) and (2) has a solution 
$u=u(t)$ if and only if $u$ is a fixed point of $T$.

Lemma 3.2. $T: P \rightarrow P$ defined by (3) is completely continuous.

Proof. From the definition of $T$, we deduce that for each $u \in P$, there is $T u \in C^{1}[0,1]$

is nonnegative and satisfies (2).

Moreover, $(\mathrm{Tu})(\sigma)$ is the maximum value of $\mathrm{Tu}$ on $[0,1]$, since

$$
(T u)^{\prime}(t)= \begin{cases}\phi_{p}^{-1}\left(\int_{t}^{\sigma} f\left(r, u(r), u^{\prime}(r)\right) d r,\right. & 0 \leq t \leq \sigma, \\ -\phi_{p}^{-1}\left(\int_{\sigma}^{t} f\left(r, u(r), u^{\prime}(r)\right) d r,\right. & \sigma \leq t \leq 1 .\end{cases}
$$

is continuous and nonincreasing in $[0,1]$ and $(T u)^{\prime}(\sigma)=0$. As $(T u)^{\prime}$ is nonincreasing on $[0,1]$, we have $T u \in P$.

Hence, we get that $T: P \rightarrow P$.

Then according to Arzela-Ascoli theorem, $T$ is completely continuous if and only if $T$ is continuous about $u$ and maps a bounded subset of $P$ into a relatively compact set.

Let $u_{n} \rightarrow u$ as $n \rightarrow \infty$ on $P$.

For $0 \leq t \leq 1$, according to (3) and (4), we have

$$
\left|\left(T u_{n}\right)(t)-(T u)(t)\right| \rightarrow 0 \quad \text { as } n \rightarrow \infty,
$$

and

$$
\left|\left(T u_{n}\right)^{\prime}(t)-(T u)^{\prime}(t)\right| \rightarrow 0 \quad \text { as } n \rightarrow \infty,
$$

Hence, we obtain that $T$ is continuous.

Now, let $\Omega \subset P$ be a bounded set, i.e., there exists a positive constant $R$ such that

$\Omega \subset\{u \in P:\|u\| \leq R\}$, for all $u \in \Omega$,

from the expression of $T u$ and $(T u)^{\prime}$ we can obtain that $T \Omega$ is uniformly bounded according to the properties of $f$. And it is also easy to get that, for any $u \in \Omega, t_{1}, t_{2} \in[0,1]$, we have

$$
\begin{array}{lll}
\left|(T u)\left(t_{1}\right)-(T u)\left(t_{2}\right)\right| \rightarrow 0, & \text { as } & t_{1} \rightarrow t_{2} . \\
\left|(T u)^{\prime}\left(t_{1}\right)-(T u)^{\prime}\left(t_{2}\right)\right| \rightarrow 0, & \text { as } & t_{1} \rightarrow t_{2} .
\end{array}
$$

which shows that $T \Omega$ is equicontinuous.

Then the Arzela-Ascoli theorem guarantees that $T \Omega$ is relatively compact, which means $T$ is compact. Then, we obtain that $T$ is completely continuous.

Thus, from what has been discussed above, we can draw the conclusion that $T: P \rightarrow P$ is completely continuous.

We are now ready to apply the fixed point theorem due to Avery and Peterson to the operator $T$ in order to get sufficient conditions for the existence of multiple positive solutions to the problems (1) and (2).

Our main result is as follows.

Theorem 3.1. Assume that there exist constants $r_{2} \geq k b>b>r_{1}>0$, $L_{2} \geq L_{1}>0$, such that $\frac{b}{M} \leq \min \left\{\frac{r_{2}}{N}, L_{2}\right\}$. If the following assumptions hold 


$$
\begin{aligned}
& \left(H_{1}\right): f(t, \omega, v)<\min \left\{\phi_{p}\left(\frac{r_{1}}{N}\right), \phi_{p}\left(L_{1}\right)\right\},(t, \omega, v) \in[0,1] \times\left[0, r_{1}\right] \times\left[-L_{1}, L_{1}\right] ; \\
& \left(H_{2}\right): f(t, \omega, v)>\phi_{p}\left(\frac{k b}{M}\right),(t, \omega, v) \in\left[\frac{1}{k}, 1-\frac{1}{k}\right] \times[b, k b] \times\left[-L_{2}, L_{2}\right] ; \\
& \left(H_{3}\right): f(t, \omega, v) \leq \min \left\{\phi_{p}\left(\frac{r_{2}}{N}\right), \phi_{p}\left(L_{2}\right)\right\},(t, \omega, v) \in[0,1] \times\left[0, r_{2}\right] \times\left[-L_{2}, L_{2}\right] .
\end{aligned}
$$

Then the boundary value problem (1) and (2) has at least three positive solutions $u_{1}, u_{2}$ and $u_{3}$ such that

$$
\begin{array}{ll}
\max _{0 \leq t \leq 1} u_{1}(t)<r_{1}, & \max _{0 \leq t \leq 1}\left|u_{1}^{\prime}(t)\right|<L_{1}, \\
b \leq \min _{1 / k \leq t \leq 1-(1 / k)} u_{2}(t) \leq \max _{0 \leq t \leq 1} u_{2}(t) \leq r_{2}, & \max _{0 \leq t \leq 1}\left|u_{2}^{\prime}(t)\right| \leq L_{2}, \\
\max _{0 \leq t \leq 1} u_{3}(t) \leq \frac{b}{\eta}, \min _{1 / k \leq t \leq 1-(1 / k)} u_{3}(t) \leq b, & \max _{0 \leq t \leq 1}\left|u_{3}^{\prime}(t)\right| \leq L_{2} .
\end{array}
$$

Proof. From we discussed earlier, E, $P, T$ is well defined. Problem (1) and (2) has a solution $u=u(t)$ if and only if $u$ is a fixed point of $T$.

We have already showed that $T: P \rightarrow P$ is completely continuous.

In what following, we will prove the results step by step according to the Theorem 2.1.

The proof is divided into some steps.

Firstly, we will show that condition $\left(H_{3}\right)$ implies that

$$
T: \bar{P}\left(\alpha, r_{2} ; \beta, L_{2}\right) \rightarrow \bar{P}\left(\alpha, r_{2} ; \beta, L_{2}\right) .
$$

In fact, for $u \in \bar{P}\left(\alpha, r_{2} ; \beta, L_{2}\right)$, we have

$$
\alpha(u)=\max _{0 \leq t \leq 1}|u(t)| \leq r_{2}, \quad \beta(u)=\max _{0 \leq t \leq 1}\left|u^{\prime}(t)\right| \leq L_{2},
$$

and assumption $\left(H_{3}\right)$ implies

$$
f\left(t, u(t), u^{\prime}(t)\right) \leq \min \left\{\phi_{p}\left(\frac{r_{2}}{N}\right), \phi_{p}\left(L_{2}\right)\right\}, \quad 0 \leq t \leq 1 .
$$

Consequently,

$$
\begin{aligned}
\alpha(T u) & =\max _{0 \leq t \leq 1}|(T u)(t)|=(T u)(\sigma) \\
& =\int_{0}^{\sigma} \phi_{p}^{-1}\left(\int_{s}^{\sigma} f\left(r, u(r), u^{\prime}(r)\right) d r\right) d s \\
& =\int_{0}^{\eta} \phi_{p}^{-1}\left(\int_{s}^{\sigma} f\left(r, u(r), u^{\prime}(r)\right) d r\right) d s+\int_{\sigma}^{1} \phi_{p}^{-1}\left(\int_{\sigma}^{s} f\left(r, u(r), u^{\prime}(r)\right) d r\right) d s \\
& \leq \frac{r_{2}}{N} \frac{1}{2}\left[\int_{0}^{1} \phi_{p}^{-1}\left(\int_{s}^{1} d r\right) d s+\int_{0}^{\eta} \phi_{p}^{-1}\left(\int_{s}^{1} d r\right) d s+\int_{\eta}^{1} \phi_{p}^{-1}\left(\int_{\eta}^{s} d r\right) d s\right] \\
& \leq \frac{r_{2}}{N} \frac{p-1}{p}=r_{2} .
\end{aligned}
$$

And by Lemma 3.1 and (4), we have

$$
\begin{aligned}
\beta(T u) & =\max _{0 \leq t \leq 1}\left|(T u)^{\prime}(t)\right| \\
& =\max \left\{(T u)^{\prime}(0),-(T u)^{\prime}(1)\right\} \\
& =\max \left\{\phi_{p}^{-1}\left(\int_{0}^{\sigma} f\left(r, u(r), u^{\prime}(r)\right) d r\right), \phi_{p}^{-1}\left(\int_{\sigma}^{1} f\left(r, u(r), u^{\prime}(r)\right) d r\right)\right\} \\
& \leq \max \left\{\phi _ { p } ^ { - 1 } \left(\int_{0}^{1} d r \phi_{p}\left(L_{2}\right), \phi_{p}^{-1}\left(\int_{0}^{1} d r \phi_{p}\left(L_{2}\right)\right\}\right.\right. \\
& =L_{2} .
\end{aligned}
$$


Then, $T: \bar{P}\left(\alpha, r_{2} ; \beta, L_{2}\right) \rightarrow \bar{P}\left(\alpha, r_{2} ; \beta, L_{2}\right)$ holds.

Secondly, we show that condition $\left(C_{1}\right)$ in Theorem 2.1 holds.

In order to check condition $\left(C_{1}\right)$ in Theorem 2.1, we choose $u(t)=k b, 0 \leq t \leq 1$.

It is easy to see that $u(t)=k b \in \bar{P}\left(\alpha, k b ; \beta, L_{2} ; \psi, b\right)$ and $\psi(u)=\psi(k b)>b$, consequently,

$$
\left\{u \in \bar{P}\left(\alpha, k b ; \beta, L_{2} ; \psi, b\right) \mid \psi(y)>b\right\} \neq \varnothing .
$$

If $u \in \bar{P}\left(\alpha, k b ; \beta, L_{2} ; \psi, b\right)$, then $b \leq u(t) \leq k b$ for $\frac{1}{k} \leq t \leq 1-\frac{1}{k}$.

From assumption $\left(\mathrm{H}_{2}\right)$, we have

$$
f\left(t, u(t), u^{\prime}(t)\right)>\phi_{p}\left(\frac{k b}{M}\right), \frac{1}{k} \leq t \leq 1-\frac{1}{k} .
$$

Then we have

$$
\begin{aligned}
\psi(T u) & =\min _{1 / k \leq t \leq 1-(1 / k)}|(T u)(t)| \geq \frac{1}{k} \max _{0 \leq t \leq 1}|(T u)(t)|=\frac{1}{k} T u(\sigma) \\
& =\frac{1}{k} \int_{0}^{\sigma} \phi_{p}^{-1}\left(\int_{s}^{\sigma} f\left(r, u(r), u^{\prime}(r)\right) d r\right) d s \\
& =\frac{1}{k} \int_{0}^{\eta} \phi_{p}^{-1}\left(\int_{s}^{\sigma} f\left(r, u(r), u^{\prime}(r)\right) d r\right) d s+\frac{1}{k} \int_{\sigma}^{1} \phi_{p}^{-1}\left(\int_{\sigma}^{s} f\left(r, u(r), u^{\prime}(r)\right) d r\right) d s \\
& \geq \frac{1}{k} \min \left\{\int_{\frac{1}{k}}^{\frac{1+\eta}{2}} \phi_{p}^{-1}\left(\int_{s}^{\frac{1+\eta}{2}} f\left(r, u(r), u^{\prime}(r)\right) d r\right) d s,\right. \\
& >\frac{b}{M} \frac{p-1}{p} \min \left\{\left(\frac{1+\eta}{2}-\frac{1}{k}\right)^{\frac{p}{p-1}},\left(\eta-\frac{1}{k}\right)^{\frac{p}{p-1}}+\left(1-\frac{1}{k}-\frac{1+\eta}{2}\right)^{\frac{p}{p-1}}\right\} \\
& =b .
\end{aligned}
$$

Then, we can get that

$$
\psi(T y)>b, \text { for all } u \in \bar{P}\left(\alpha, k b ; \beta, L_{2} ; \psi, b\right) .
$$

Consequently, condition $\left(C_{1}\right)$ in Theorem 2.1 holds.

Thirdly, We now show $\left(C_{2}\right)$ in Theorem 2.1 is satisfied.

If $u \in \bar{P}\left(\alpha, r_{1} ; \beta, L_{1}\right)$, then assumption $\left(H_{1}\right)$ yields

$$
f\left(t, u(t), u^{\prime}(t)\right)<\min \left\{\frac{r_{1}}{N}, L_{1}\right\}, 0 \leq t \leq 1 .
$$

In the same way as in the first step, we can obtain that $T: \bar{P}\left(\alpha, r_{1} ; \beta, L_{1}\right) \rightarrow \bar{P}\left(\alpha, r_{1} ; \beta, L_{1}\right)$. Hence, condition $\left(C_{2}\right)$ in Theorem 2.1 is aslo satisfied.

Finally, we show $\left(C_{3}\right)$ in Theorem 2.1 is also satisfied.

Suppose that $u \in \bar{P}\left(\alpha, r_{2} ; \beta, L_{2} ; \psi, b\right)$ with $\alpha(T u)>k b$. Then, by the definition of $\psi$ and $T u \in P$, we have

$$
\psi(T u)=\min _{1 / k \leq t \leq 1-(1 / k)}|(T u)(t)| \geq \frac{1}{k} \max _{0 \leq t \leq 1}|(T u)(t)|=\frac{1}{k} \alpha(T u)>b .
$$


Thus, condition $\left(C_{3}\right)$ in Theorem 2.1 is also satisfied.

Then, Theorem 3.1 is proved by Theorem 2.1 .

Consequently, Theorem 3.1 is proved by Theorem 2.1. We obtain that the boundary value problem (1) and (2) has at least three positive solutions $u_{1}, u_{2}$ and $u_{3}$ such that

$$
\begin{aligned}
u_{1} & \in P\left(\alpha, r_{1} ; \beta, L_{1}\right), \\
& u_{2} \in\left\{\bar{P}\left(\alpha, r_{2} ; \beta, L_{2} ; \psi, b\right) \mid \psi(y)>b\right\}, \\
\text { and } \quad u_{3} & \in \bar{P}\left(\alpha, r_{2} ; \beta, L_{2}\right) \backslash\left(\bar{P}\left(\alpha, r_{2} ; \beta, L_{2} ; \psi, b\right) \cup \bar{P}\left(\alpha, r_{1} ; \beta, L_{1}\right)\right) .
\end{aligned}
$$

\section{Acknowledgements}

The author thanks the referees for their valuable comments and suggestions. This work was supported by the Discipline Construction Fund of Central University of Finance and Economics.

\section{References}

[1] II'in, V. and Moiseev, E. (1987) Nonlocal Boundary Value Problem of the Second Kind for a Sturm-Liouville Operator. Diff. Eqs., 23, 979-987.

[2] He, X. and Ge, W. (2002) Multiple Positive Solutions for One-Dimensional p-Laplacian Boundary Value Problem. Appl.Math.Lett., 15, 937-943. https://doi.org/10.1016/S0893-9659(02)00067-8

[3] Sun, B., Ge, W. and Zhao, D. (2007) Three Positive Solutions for Multipoint One-Dimensional p-Laplacian Boundary Value Problems with Dependence on the First Order Derivative. Math. Comput. Model., 45, 1170-1178. https://doi.org/10.1016/j.mcm.2006.10.002

[4] Ma, R. (2001) Positive Solutions for Second Order Three-Point Boundary Value Problems. Appl.Math.Lett., 14, 1-5. https://doi.org/10.1016/S0893-9659(00)00102-6

[5] Gupta, C. (1998) A Generalized Multipoint Boundary Value Problem for Second Order Ordinary Differential Equations. Appl. Math. Comput., 89, 133-146. https://doi.org/10.1016/S0096-3003(97)81653-0

[6] Wang, J. and Zheng, D. (1997) On the Existence of Positive Solutions to a Three-Point Boundary Value Problem for the One-Dimensional p-Laplacian. ZAMM., 77, 477-479. https://doi.org/10.1002/zamm.19970770618

[7] Ma, R. and Castaneda, N. (2001) Existence of Solutions of Nonlinear M-Point Boundary Value Problems. J. Math. Anal. Appl., 256, 556-567. https://doi.org/10.1006/jmaa.2000.7320

[8] Jiang, D., Nieto, J.J. and Zuo, W. (2004) On Monotone Method for First Order and Second Order Periodic Boundary Value Problems and Periodic Solutions of Functional Differential Equations. J. Math. Anal. Appl., 289, 691-699. https://doi.org/10.1016/j.jmaa.2003.09.020

[9] Ahmad, B. and Sivasundaram, S. (2006) The Monotone Iterative Technique for Impulsive Hybrid Set Valued Integro-Differential Equations. Nonlinear Anal., 65, 2260-2276. https://doi.org/10.1016/j.na.2006.01.033

[10] Bai, Z., Ge, W. and Wang, Y. (2005) Multiplicity Results for Some Second-Order Four-Point Boundary Value Problems. Nonlinear Anal., 60, 491-500. 